\title{
Impacto del paisaje sonoro urbano desde el registro subjetivo de los usuarios. Abordaje metodológico- instrumental
}

\author{
Impact of the urban soundscape from the subjective \\ register of the users. Methodological-instrumental \\ approach
}

\section{Resumen}

Autores:

Marta Susana Cisterna* mcisterna@herrera.unt.edu.ar

Arturo Raúl Maristany** amaristany@unc.edu.ar

Guillermo Enrique Gonzalo* ggonzalo@herrera.unt.edu.ar

*Universidad Nacional de Tucumán ** Universidad Nacional de Córdoba

Argentina

Recibido: 12/Nov/2020 Aceptado: 25/May/2021 e desarrolla un análisis que integra el enfoque objetivo de la acústica con el enfoque subjetivo del concepto paisaje sonoro. A través de una metodología cuanti-cualitativa se analiza el sonido en sí mismo, junto a la valoración de los sujetos que lo perciben, situados en determinado contexto ambiental, social y cultural. El objeto de estudio elegido es la Plaza Urquiza de Tucumán, Argentina, de valor histórico patrimonial, donde se desarrollan tradicionalmente actividades de esparcimiento. La metodología plantea el relevamiento de condiciones físicas, niveles de presión sonora, ubicación y estructura temporal de fuentes de sonido. Luego, con el fin de conocer la percepción y valoración del paisaje sonoro, propone realizar entrevistas y encuestas, cuyos datos son procesados por estadística descriptiva y Método Comparativo Constante. La investigación desarrolla una metodología factible de ser aplicada para el estudio del paisaje sonoro y permite trazar recomendaciones de diseño para mejorar la calidad acústica de este espacio público y de otros similares.

Palabras clave: paisaje sonoro; metodología de estudio; valoración subjetiva; espacio urbano; Tucumán.

\section{Abstract:}

An analysis is developed which integrates the objective approach to acoustics with the subjective approach to the concept of soundscape. Through a quantitativequalitative methodology, the sound itself is analyzed, as well as the evaluation of the subjects who perceive it, located in a certain environmental, social and cultural context. The chosen object of study is the Plaza Urquiza in Tucumán, Argentina, of historical heritage value, where leisure activities are traditionally developed. The methodology involves the study of the physical conditions, sound pressure levels, location and temporal structure of sound sources. Then, in order to know the perception and the evaluation of the soundscape, he proposes to carry out interviews and surveys whose data are processed by descriptive statistics and the constant comparison method. The research develops a feasible methodology to be applied for the study of the soundscape and allows the conception of design recommendations to improve the acoustic quality of this public space and others like it.

Keywords: soundscape; study methodology; subjective assessment; urban space; Tucumán. 


\section{Introducción}

El presente trabajo constituye un avance de la tesis doctoral: "El paisaje sonoro y la interacción de colectivos sociales en el espacio público urbano de Tucumán. Abordaje metodológicoinstrumental", de la carrera de Doctorado en Arquitectura, que se dicta en la Facultad de Arquitectura y Urbanismo de la Universidad Nacional de Tucumán. Se propone enfocar el concepto de paisaje sonoro tal como lo aborda el análisis de la acústica de espacios urbanos, pero se desarrolla desde una perspectiva integradora del estudio de la física del sonido conjuntamente con el análisis de los aspectos humanos subjetivos que inciden en su valoración. Para llevar a cabo este objetivo propone la aplicación de una metodología complementaria cuanticualitativa que posibilita focalizar el estudio del sonido al mismo tiempo que la valoración del impacto en la subjetividad de los sujetos que lo perciben, situándolos en determinado contexto ambiental, social y cultural. El objeto de estudio aquí propuesto es la plaza Urquiza de Tucumán, importante enclave urbano, de valor histórico patrimonial, donde tradicionalmente se desarrollan actividades de esparcimiento, deportivas, culturales y comerciales. La plaza convoca a personas con distintas motivaciones, que concurren desde otros sectores de la ciudad y localidades del interior de la Provincia para disfrutar de todo lo que este espacio les brinda. E interés suscitado por el paisaje sonoro que allí se genera, se relaciona con la riqueza que presenta en cuanto a las fuentes sonoras, las actividades y los cambios que se perciben durante el día y en el transcurso de la semana. Con el fin de conocer la valoración subjetiva, la investigación plantea un análisis cuantitativo sobre la estructura temporal, frecuencia y niveles sonoros físicos. Al mismo tiempo, propone articular dichas características con el impacto que las mismas tienen en la subjetividad de los usuarios. Para alcanzar este objetivo se aplican técnicas propuestas por la metodología complementaria cuanti-cualitativa, que permiten ver efectos diferenciables del estímulo sonoro en las personas, en articulación con otros estímulos, que llegan en sincronía e inciden conjuntamente.

\subsection{La ciudad y la plaza en sus orígenes}

San Miguel de Tucumán, desde sus orígenes, presenta una conformación urbana con un polo o centro, alrededor del cual se desarrollaron anillos concéntricos que pueden identificarse con las diferentes etapas de crecimiento. El trazado original, con aceras angostas, carentes de arbolado, con la Plaza Independencia en su centro, se reconoce aún hoy en la ciudad y se denomina Casco Fundacional (Paterlini, 2010). Esta configuración se mantuvo hasta el siglo XIX, cuando se suma el Trazado del Período Liberal, que presenta como rasgos característicos calles y veredas de mayor dimensión, arboladas, con la incorporación de otras plazas y el anillo de avenidas o bulevares. Adyacente al Casco Liberal se encuentra el Parque del Centenario, hoy "9 de Julio". El conjunto se reconoce desde 1930 como Área Central. Modificada actualmente por la edificación en altura, presenta el desarrollo del espacio público como trama regular, definido por una edificación continua y por calles-canal (Di Lullo y Giobellina, 1996), morfología que incide en el entorno sonoro, con prevalencia del tránsito vehicular como ruido de fondo de la ciudad. Las zonas de esparcimiento, como las plazas y el parque, presentan características sonoras diferentes al permitir la presencia de sonidos naturales, como el canto de los pájaros o el sonido del agua.

En el Área Central de la ciudad se encuentra la Plaza Urquiza, rodeada de importantes edificios de valor histórico patrimonial. En los últimos años se han construido en su entorno edificios de gran altura, al mismo tiempo que la plaza se ha transformado en un lugar de actividades muy diversas: manifestaciones ciudadanas, ferias de artesanías, muestras escolares, clases de baile, clases de gimnasia, ferias y venta de diversos productos, muchas de estas actividades organizadas desde el gobierno y la municipalidad de San Miguel de Tucumán.

Del análisis de textos que describen cómo era la zona de la plaza a principios del siglo pasado, se desprende que la misma presentaba características suburbanas con cañaverales y pequeñas quintas de citrus en las adyacencias. En sus orígenes contaba con características de espacio verde, con prevalencia de un ambiente natural, y contaba con un lago artificial donde se realizaban paseos en botes a remo. En la plaza eran frecuentes los espectáculos al aire libre, como las tradicionales fiestas de la primavera. La mayor parte de su superficie estaba cubierta de verde con senderos empedrados o adoquinados, una importante arboleda, pérgolas y plantas con flores.

La plaza conservó su carácter original hasta la remodelación del año 1979, a cargo del arquitecto Ricardo Salim. El pequeño lago se reemplazó por uno artificial que recorre parte de la plaza, se agregaron caminerías y luminarias, también un sector de juegos para niños y equipamiento como bancos y bebederos, se mantuvieron la mayoría de los árboles, incluido el "gomero", de gran porte, que se encuentra en su sector central, en el cruce de las diagonales. Desde entonces se han realizado modificaciones, pero estas características se han mantenido hasta el presente.

\subsection{El concepto de paisaje sonoro}

El concepto de paisaje sonoro cuenta con importantes investigaciones y propuestas metodológicas que constituyen un valioso aporte. Se mencionan como antecedentes sobre el tema algunos resultados alcanzados en ciudades con diversas culturas y diferentes ambientes sonoros.

Se considera importante iniciar esta reseña señalando que, a mediados del siglo pasado, científicos e investigadores comenzaron a plantear la importancia de los sonidos y su incidencia en la salud humana. En 1966 Pierre Schaeffer 
-compositor francés- publicó su "Tratado sobre objetos musicales", en el que propone un nuevo vocabulario sobre el sonido. En su obra plantea cuatro ejes o formas de audición: escuchar, oirr, entender y comprender. Define escuchar y oír como formas concretas, diferenciándolas como objetiva y subjetiva respectivamente. Señala también que entender y comprender son ejes abstractos, subjetivo y objetivo, respectivamente (Schaeffer, 2003). Unos años más tarde, en 1968, Raymond Murray Schafer -compositor canadiense- publicó su libro "The New Soundscape", donde destaca la importancia del entorno acústico en la vida cotidiana. Con el término paisaje sonoro, Schafer se refiere a los sonidos producidos en un espacio determinado, con una lógica o sentido otorgado por el entorno social en el que se producen, e indica la evolución de dicho entorno o sociedad. Propone la distinción entre ambientes sonoros de alta fidelidad "hi-fi" y de baja fidelidad, "Io-fi". En los de alta fidelidad, los sonidos no se superponen, tienen perspectiva, primer plano y fondo; por el contrario, en los ambientes de baja fidelidad, los planos compositivos se cubren y enmascaran, resultando difícil discernir entre figura y fondo (Schafer, 1977). El concepto de paisaje sonoro fue difundido en Europa, principalmente por el proyecto New Soundscape Project -Proyecto del nuevo paisaje sonoro-, y a través de investigaciones interdisciplinarias, relacionadas con el sonido en entornos rurales o urbanos (Schafer, 1976). A partir de sus estudios comenzaron a sumarse numerosas investigaciones sobre los efectos del sonido en diversos entornos con enfoques $y$ resultados acordes a diferentes culturas. En Europa, el filósofo, urbanista y musicólogo francés, Augoyard, cofundador del Centre de recherche sur l'espace sonore et l'environnement urbain-Centro de investigación sobre espacio sonoro y entorno urbano- CRESSON, desde 1979, investiga los efectos sonoros en el medio ambiente urbano, el paisaje, el espacio público y las relaciones entre el arte y la ciudad. El Foro Mundial para la Ecología Acústica de 1993, estableció la aceptación del concepto de paisaje sonoro a nivel mundial. La contribución del mismo se basa en comparar las características físicas de los entornos urbanos con la conciencia perceptiva de sus habitantes y usuarios. A partir de estos avances es posible destacar una importante producción de investigadores y teóricos que abordaron el tema de paisaje sonoro con diversos enfoques y desarrollaron investigaciones donde analizan el ambiente sonoro desde un punto de vista integral, donde el sonido no es entendido como un mero elemento físico del medio, sino como un elemento de comunicación e información entre las personas y el medio urbano (García, 2010; Germán González y Santillán, 2006; Maristany, 2016; Zapata Cardona y Cardona Restrepo, 2020). Otros autores (Botteldooren, De Coensel y De Muer, 2006; López Barrio, 2001) han investigado la contaminación acústica en la ciudad y la ecología acústica, la relación o correspondencia entre la contaminación acústica y el paisaje sonoro. Sus trabajos presentan avances en el estudio de indicadores que permiten evaluar, de manera objetiva, la calidad de los paisajes sonoros urbanos. Señalan también la necesidad de análisis adicionales de la relación entre los indicadores objetivos y evaluaciones subjetivas de la calidad del sonido urbano, por parte de un participante activo. Otra línea actual de investigación explora las asociaciones entre paisaje sonoro y bienestar in situ, con aplicación de cuestionarios en diferentes entornos. Los resultados muestran que existen asociaciones entre la percepción de paisajes sonoros, la salud y el bienestar individual (Aletta et al., 2019).

\section{Métodos}

Se optó por trabajar con una metodología complementaria cuanti-cualitativa que permite articular el análisis de las características físicas de los entornos urbanos con la conciencia perceptiva de sus habitantes y usuarios. Las técnicas aplicadas permitieron conocer los registros y la valoración de los sonidos percibidos por las personas, situadas en un contexto urbano concreto. La metodología propuesta se fundamenta en la denominada "Grounded Theory", desarrollada por Glaser y Strauss (1967), traducida como "Teoría Fundamentada" o "Teoría de Base", y se refiere a una teoría que se construye a partir de los datos obtenidos en relevamientos empíricos, los cuales derivan en conclusiones, a través de procesos inferenciales inductivos. En relación a esta metodología, Hernández Sampieri y Mendoza destacan que la esencia de la investigación cualitativa consiste en comprender los fenómenos, explorándolos desde la perspectiva de los participantes, en su ambiente natural, y en relación con el contexto. Señalan una serie de recomendaciones entre las que incluyen el apoyo con datos cuantitativos (Hernández Sampieri y Mendoza, 2018).

Como expresa la socióloga Kathleen Charmaz, los métodos de teoría fundamentada consisten en pautas sistemáticas, pero flexibles, para recopilar y analizar datos cualitativos que permiten construir teorías "fundamentadas" en los datos mismos. Señala que la codificación permite filtrar los datos, ordenarlos y nos da control para hacer comparaciones con otros segmentos de datos. Al codificar y realizar numerosas comparaciones, nuestra comprensión analítica de los datos comienza a tomar forma. Las notas analíticas preliminares -memorandosy las comparaciones, permitirán definir las ideas que mejor se ajustan e interpretan los datos como categorías analíticas provisionales Charmaz (2006).

En nuestra investigación, la codificación y análisis de los datos se basaron en el método comparativo constante, el cual plantea el cotejo continuo de argumentos específicos y de datos obtenidos en el análisis de textos históricos, entrevistas, notas de campo de observaciones y encuestas, con el fin de ir refinando paulatinamente los conceptos, identificando sus propiedades, explorando sus interrelaciones e integrándolos en una teoría. A medida que los conceptos fueron identificados y se comenzó a desarrollar la teoría, se pudo observar que, en ciertos momentos, la investigación necesitó incorporar más datos procedentes del colectivo investigado. Esta ampliación de la muestra hasta el punto que llamamos "saturación", nos permitió encontrar fundamentos más sólidos, los cuales forman parte de este trabajo. Al respecto, Matthew, Miles, Huberman y Saldaña (2019) señalan que los datos codificados, comparados y elaborados, permiten generar el corpus empírico de la investigación sobre el cual se fundamentará la teoría.

En el estudio del paisaje sonoro de la plaza, los datos obtenidos a través de las observaciones y de las 
técnicas de relevamiento, dieron lugar al surgimiento de significados sobre el fenómeno de percepción de sonido. A partir de los datos empíricos aportados por quienes concurren al lugar, se logró desarrollar teoría sobre el impacto de los sonidos en la subjetividad. En las primeras visitas al terreno se utilizó la técnica de observación borrosa o "difusa", la cual consiste en visitas al terreno con el fin de observar sin enfocarse en nada particular, brindando igual atención a las personas y al contexto. En las siguientes visitas se realizó una forma de observación que se utiliza frecuentemente en la investigación cualitativa, la observación participante. Denzin (1989) define la observación participante como una estrategia de campo que combina simultáneamente el análisis de documentos, la entrevista a informantes, la participación directa, la observación, y la introspección (Citado en Flick, 2007, p. 154).

Las observaciones y relevamientos se realizaron durante el año 2019 hasta marzo del 2020, en diferentes meses y días de semana. En función de las primeras observaciones, se decidió tomar mediciones, realizar entrevistas y encuestas durante los fines de semana, en el horario de 18:00 h a 22:00 h, para registrar as las situaciones de mayor concurrencia en la plaza, las actividades, la cantidad de personas y las fuentes sonoras presentes, y conocer cómo inciden en la generación de los paisajes sonoros. Se presentan aquí los resultados de las observaciones y entrevistas realizadas durante un año, y las encuestas y mediciones realizadas en los meses de marzo y abril de 2019, y en febrero y marzo de 2020.

Por un lado, se analizaron los sonidos como estímulos físicos que provienen de diferentes fuentes: automóviles, música de los juegos infantiles -principalmente la calesita-, música de las clases de gimnasia y de los grupos de jóvenes, conversaciones, voces de vendedores ambulantes, ladridos de las mascotas, etc. Los sonidos fueron registrados con un sonómetro, ubicado en diferentes puntos, con el fin de captar las fuentes sonoras presentes en la plaza. Sincrónicamente se propuso conocer la articulación entre los sonidos y las vivencias de alegría o desagrado suscitadas en los usuarios. Para ese relevamiento se realizaron entrevistas libres y entrevistas en profundidad a informantes clave y se diseñó un cuestionario de preguntas cerradas y abiertas, cuyos resultados se presentan a continuación. Luego, a través del método comparativo constante, se analizaron las expresiones de los usuarios para extraer datos y codificarlos. Se agruparon las transcripciones en categorías, temas o conceptos con el fin de establecer relaciones, encontrar coincidencias y aportar a las conclusiones.

Los datos obtenidos en las encuestas se procesaron través de estadística descriptiva con el fin de elaborar conclusiones sobre el paisaje sonoro, con articulación de los sonidos y la valoración subjetiva de los usuarios. A través de los procesos de análisis y razonamiento inferencial inductivo se llegó a conclusiones sobre la metodología aplicada y el paisaje sonoro analizado, y se enunciaron también posibles estrategias de diseño para mejorar la calidad acústica de este y otros espacios públicos similares.

\subsection{Instrumentos y técnicas aplicadas}

Con el fin de caracterizar el ambiente sonoro de la plaza se realizaron las primeras visitas al lugar con la técnica de observación borrosa, que consiste en salidas al campo para descubrir lo que sucede. Luego, se realizaron relevamientos de las condiciones físicas y acústicas a través de mediciones y de la valoración de los usuarios, con encuestas y entrevistas (Figura 1)

Para las mediciones del nivel de presión sonora de la plaza en diferentes días y horarios, se utilizó un sonómetro, ubicado en sectores determinados. De forma sincrónica, se realizaron entrevistas y encuestas tipo cuestionario, con preguntas cerradas y abiertas. La encuesta primeramente solicita algunos datos personales y luego indaga sobre los motivos de elección de la plaza, frecuencia con que concurren, preferencia de algún sector o lugar, y los motivos para elegirlo. Indaga específicamente sobre los sonidos percibidos en el lugar sobre los sentimientos de molestia o de agrado que les producen en forma abierta interroga sobre los aspectos que les gustaría modificar en la plaza en general, y en particular, sobre las fuentes sonoras mencionadas. Se realizaron también entrevistas que permitieron profundizar sobre la valoración del paisaje sonoro, las vivencias suscitadas por los sonidos y sobre la percepción y valoración de otros estímulos presentes.

El proceso de recopilación de datos se basó en el "muestreo teórico" desarrollado por Glaser y Strauss. Los autores señalan que, en este proceso, el investigador recoge, codifica y analiza conjuntamente sus datos, y decide qué datos recopilar a continuación y dónde buscarlos, a fin de desarrollar su teoría. El proceso está controlado por la teoría emergente, ya sea sustantiva o formal. En el muestreo teórico, dirigido a la construcción de teoría, todo informante, usuario entrevistado o encuestado, tiene el mismo peso para la investigación (Glaser y Strauss, 1967, p. 45)

Además de las observaciones y entrevistas, se realizaron 59 encuestas a sujetos que se encontraban realizando distintas actividades en los sectores que integran la plaza. Las encuestas se realizaron en los meses de marzo y abril de 2019, y en febrero y marzo de 2020, durante los fines de semana. Fueron encuestadas 34 mujeres y 25 varones, con rango etario entre 12 y 69 años, de diversas ocupaciones: estudiantes, amas de casa, constructor, empleados, ingeniero, arquitectos, docentes, desempleados y jubilados. En el cuestionario se solicitan datos personales como el lugar o barrio de residencia: la mayoría de los encuestados (82\%) declaró vivir en barrios alejados, a más de veinte cuadras de la plaza; de ellos, el $45 \%$ vive en otra localidad; solo un $18 \%$ de los encuestados vive en la zona cercana, en un radio de 15 cuadras (Figura 2). 
EVALUACION DE ESPACIOS PUBLICOS DE SAN MIGUEL DE TUCUMAN

Este cuestionario forma parte de un trabajo de tesis en desarrollo en la Facultad de Arquitecturay Urbanismo, UNT. Tiene la finalidad de conocer la opinión de los usuarios de espacios públicos de Tucumán.
1. Edad:
2 - Género: 3-Ocupación:

4 - Lugar donde vives, barrio, localidad:

5- ¿Con qué frecuencia vienes a este lugar: (elige una opción)
a) Todos los días
b) Los fines de semana
c) A veces
d) Otro .

6- ¿Qué lugar o zona de esta plaza te gusta o prefieres?

7- Motivos por los cuales eliges este lugar: (puedes elegir más de una opción)

a) Me siento seguro/a

b) Me gusta la gente que frecuenta este lugar

c) Es un lugar lindo, cómodo, limpio

d) Es tranquilo, puedo charlar, relajarme, leer y hacer otras actividades

e) Puedo andar en skate, en patines o bicicleta

f) Me gusta porque hay muchos árboles y pájaros

g) Me gusta porque hay gente, hay música y muchas actividades

h) Me gusta porque es un lugar concurrido, se ve movimiento de gente y de autos

i) Vengo aquí porque no encuentro otras opciones de lugares a donde ir

j) Otros motivos

8- ¿Qué sonidos escuchas en este momento? (puedes elegir más de una opción)

a) De autos, motos, del tránsito de la calle

b) Las voces de la gente que conversa

c) Del agua de una fuente o bebedero

d) De las mascotas

e) De los niños que juegan aquí

f) De los vendedores ambulantes

g) Música del sector de juegos infantiles

h) El canto de los pájaros

i) No logro distinguir los sonidos que escucho aquí, es una mezcla y no los distingo

j) Otros

9- De los sonidos que escuchas ¿Cuáles te molestan o desagradan?

9.a - ¿Puedes decir los motivos?

10- De los sonidos que escuchas ¿Cuáles no te molestan o agradan?

10.a- ¿Puedes decir los motivos?

11. ¿Cómo te sientes en este lugar?

12. ¿Hay algo que te gustaría cambiar o modificar de este lugar?

Muchas gracias por responder la encuesta

Figura 1: Cuestionario sobre la opinión de los usuarios Fuente: M.S. Cisterna (2019) 


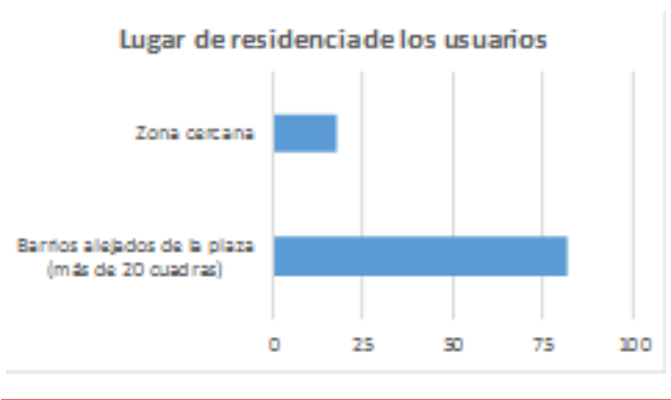

Figura 2: Lugar de procedencia de usuarios de la plaza. Fuente: Encuestas realizadas $(2019-2020)$

Las respuestas de las encuestas y entrevistas muestran que la plaza es considerada como lugar de esparcimiento a nivel provincial; esto en parte se debe a su ubicación y a la conectividad que presenta a través de las vías de circulación adyacentes, con líneas de colectivos urbanos e interurbanos, y también a las diversas actividades que allí se organizan y son difundidas por los medios de comunicación. Según el relevamiento, el lugar constituye un polo de atracción, con un radio de influencia de aproximadamente $15 \mathrm{~km}$. En las encuestas se determinó que el área del Gran San Miguel de Tucumán (GSMT: aglomerado urbano formado por la ciudad de San Miguel de Tucumán y localidades cercanas) constituye la zona de procedencia del $45 \%$ de los usuarios encuestados en la plaza (Figura 3).

La encuesta también interroga sobre la frecuencia con que asisten a la plaza. El $43 \%$ de las personas concurren principalmente los fines de semana, el $32 \%$ eventualmente para participar de actividades previamente organizadas, y el $25 \%$ concurre todos los días.

Sobre las motivaciones para elegir la plaza como lugar de esparcimiento, la respuesta que mayormente se registra, $86 \%$, menciona la tranquilidad que brinda y su abundante arboleda. Aquí se evidencia una contradicción o inconsistencia entre las expresiones de los usuarios y los niveles de sonidos relevados, principalmente en los fines de semana, cuando se registran niveles sonoros altos, con presencia de múltiples fuentes y ruido de fondo elevado. Otro aspecto mencionado (78\%) es la sensación de estar en un lugar seguro, principalmente por la presencia de policías, por su ubicación y su adecuada iluminación artificial. El $70 \%$ de las respuestas mencionan como algo positivo, la presencia de gran cantidad de personas, al igual que en las entrevistas realizadas, se valora como una característica que "...da vida a la plaza,...", hacen referencia a la percepción de un lugar dinámico relacionado con la gran concurrencia, los sonidos y la circulación de personas y de automóviles en sus veredas y calles perimetrales. En menor porcentaje (50\%), se mencionan aspectos estéticos, de comodidad y limpieza (Figura 4).

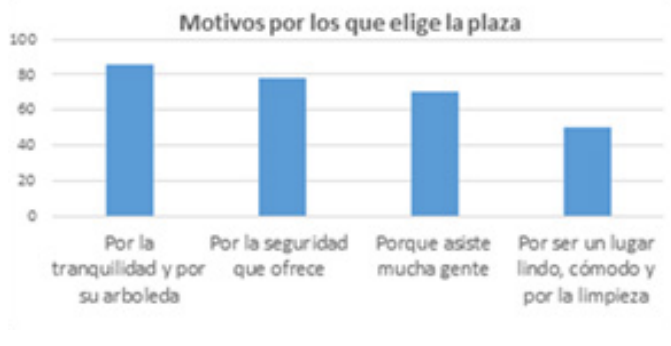

Figura 4: Motivaciones de usuarios de la plaza Fuente: Encuestas realizadas (2019 - 2020)

En relación a los sectores de la plaza que eligen, se mencionaron tres como los mayormente elegidos: el 32\% prefiere el sector central. Lo mencionan como preferido para realizar actividades de esparcimiento, o actividad física, también para escuchar música, encontrarse con amigos, o simplemente para observar lo que allí sucede. El sector de juegos infantiles es otro sector con mayor concurrencia, y el $21 \%$ lo menciona. Otros sectores,

LOCALIDADES DE GRAN SAN MIGUEL DE TUCUMÁN

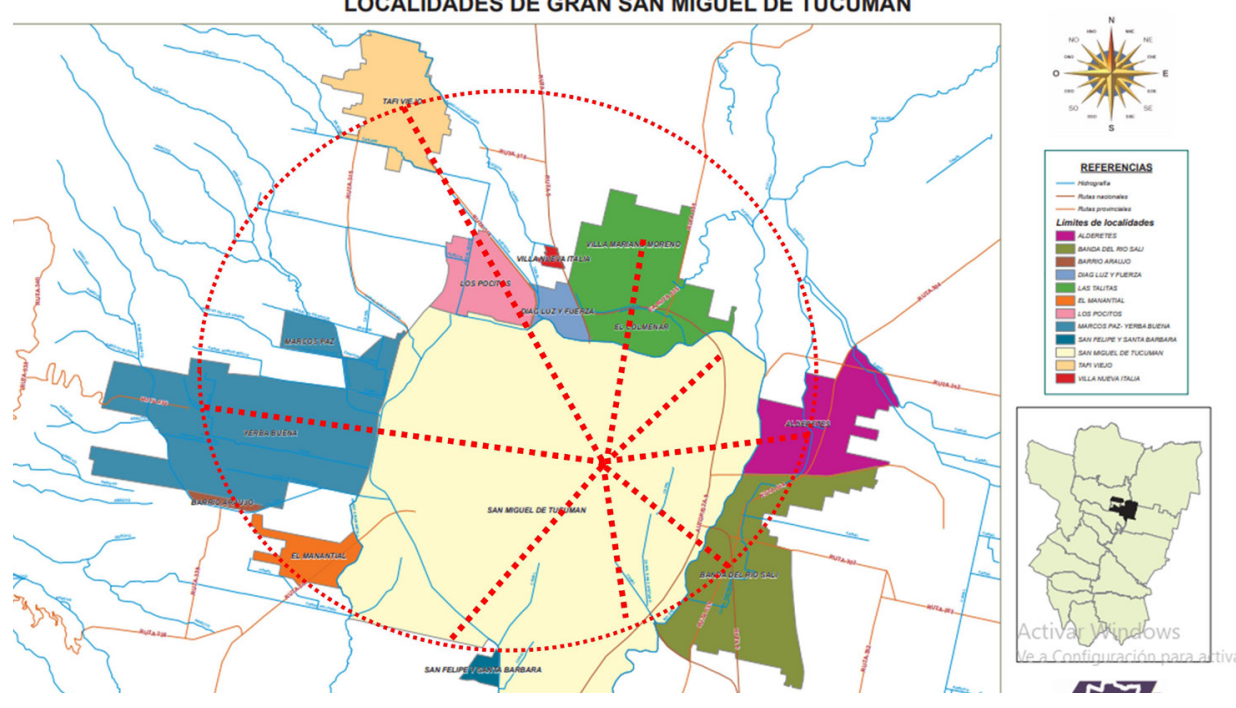

Figura 3: Mapa del GSMT con las localidades de procedencia de usuarios relevados por las encuestas Fuente: Infraestructura de datos espaciales, Provincia de Tucumán. Depto SIG (2020) 
como la explanada del mástil, que se encuentra frente a avenida Sarmiento y el sector frente al colegio Nacional B Mitre, donde se encuentra un monumento a J.L. Borges, fueron mencionados en el $19 \%$ de las encuestas. El 28\% de los usuarios manifestaron no elegir ningún lugar de la plaza en particular (Figura 5).

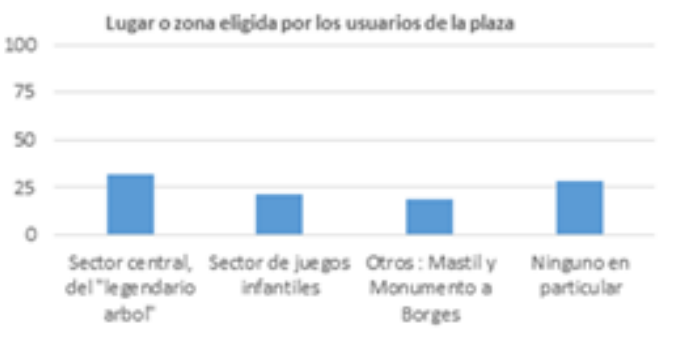

Figura 5: Lugar elegido por los usuarios de la plaza Fuente: Encuestas realizadas (2019 - 2020)

En cuanto a las fuentes, en cada uno de los sectores los usuarios mencionaron diferentes sonidos que reconocen como predominantes y característicos del lugar. Si bien el tránsito vehicular es percibido como ruido de fondo, en algunos sectores se presenta como la fuente de mayor intensidad que enmascara a los otros sonidos. Los usuarios identificaron las siguientes fuentes sonoras:

1. Tránsito vehicular (circulación de autos, motos y colectivos)

2. Música de los juegos infantiles

3. Voces de los niños

4. Música de los equipos de sonido para algunas actividades

5. Canto de pájaros

6. Ladridos de perros

En relación a los sonidos registrados por usuarios, los relevamientos muestran que en las zonas de juegos infantiles y en el centro de la plaza, donde se encuentra el legendario árbol, $89 \%$ de los usuarios mencionan la música, y $82 \%$ mencionan las voces de niños y el tránsito vehicular como las fuentes de mayor presencia. En las zonas de borde se mencionó con mayor frecuencia el tránsito vehicular como un sonido predominante, seguido por ladridos de mascotas y voces humanas percibidas en menor medida. En la zona del lago, la mayoría mencionó la música y el "ruido de la calle" (tránsito vehicular) como las principales fuentes sonoras. Con menor frecuencia (40\%) se mencionan las conversaciones, los vendedores ambulantes, los ladridos de mascotas y el canto de los pájaros (Figura 6).

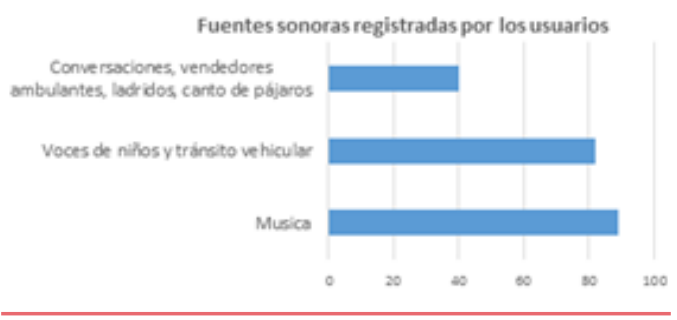

Figura 6: Fuentes sonoras registradas

Fuente: Encuestas realizadas (2019 - 2020)
Las entrevistas permitieron profundizar algunos aspectos sobre la plaza y su paisaje sonoro. La mayoría de los entrevistados coincidieron en la valoración positiva de las fuentes sonoras que perciben en la plaza; incluso al preguntárseles si desearían disminuir la intensidad de algunos sonidos, solo el 2,5\% mencionó que les gustaría disminuir en volumen de la música. Las expresiones recogidas por las encuestas fueron reafirmadas a través de los relatos de los usuarios sobre sus vivencias. Algunas personas manifestaron su preferencia por el sector cercano a los juegos infantiles y los bancos bajo las arboledas; expresaron que la risa de los niños y el canto de los pájaros les agradan, les transmite paz y los relaja.

Sobre el ruido del tránsito vehicular, los entrevistados coincidieron en que, si bien no era una fuente agradable, no les gustaría que disminuyera y consideran que es un aporte positivo a la "vida" de la plaza. Una minoría manifestó que concurren en busca de un lugar tranquilo, para conversar o leer, indicando que el lugar más apropiado es donde se encuentra el monumento a Jorge Luis Borges, en la acera norte, frente al colegio Nacional Bartolomé Mitre; hasta allí no llegan las fuentes del sector de juegos infantiles ni la música de las reuniones de jóvenes, y se observa también que es el sector con menor concurrencia de usuarios. La mayoría mencionó como positivas las actividades que se desarrollan los fines de semana: clases de Tango, gimnasia, ferias de platos, concursos de baile de ritmos modernos $y$, en algunas oportunidades, obras de teatro.

\section{Resultados}

\subsection{El paisaje sonoro en la ciudad actual}

En la ciudad actual se producen diversos sonidos que se propagan en su espacio y se integran de manera inalienable a su morfología. Sus habitantes, a la vez de producir muchos de esos sonidos, los reconocen como parte del entorno, la mayoría de las veces, sin cuestionarlos. De esta manera, el sonido de un avión en el aire, los ruidos de las actividades industriales, el tráfico de diversos tipos de vehículos, el zumbido de transformadores y de sistemas de ventilación, son sonidos reconocidos por todos y originan un ambiente ruidoso que determina una reducción considerable del bienestar de la población (De Coensel, 2007). Sin duda, los niveles sonoros ambientales han aumentado paulatinamente con el desarrollo económico y social, casi como una consecuencia inevitable. Es posible observar cuán diferente es el entorno acústico actual al descrito en el pasado por los habitantes de las ciudades. Históricamente, a partir de considerar el ruido como agente contaminante comenzaron a desarrollarse normas y leyes para su control y disminución. Sin embargo, es importante valorar que el sonido constituye un vínculo entre las personas y el medio ambiente, los sonidos impactan de manera positiva o negativa sobre la percepción e inciden en el comportamiento de los sujetos. En su obra "Handbook of Acoustic Ecology", Truax se refiere al "espacio acústico" como el perfil de un sonido, o señal de sonido, sobre su entorno circundante. El espacio acústico de cualquier sonido es el área sobre la cual es posible escucharlo, antes de que caiga 
por debajo del nivel de ruido ambiental. Señala que la disminución del área de estos espacios es consecuencia del crecimiento de los sonidos de fondo, que adquieren cada vez mayor protagonismo en los entornos urbanos (Truax, 1999).

En relación a las investigaciones sobre ambiente sonoro hace algunos años se centraban especialmente en la evaluación de la variable "intensidad del sonido", siendo el principal objetivo la reducción de las emisiones. Según este enfoque, el concepto de confort acústico se definía por un criterio cuantitativo: "a menor intensidad, mayor confort". Estudios que integran aspectos subjetivos, cualitativos, muestran que la disminución o ausencia de ruido no puede ser considerada como caracterizadora de una situación sonora perfecta. Aunque la intensidad es una variable necesaria para el análisis, por sí sola no define la respuesta ante el ambiente sonoro. En este sentido, es importante señalar que las propiedades acústicas de los espacios derivan, en gran medida, del diseño urbano, de su solución formal, dimensiones, materiales, sus texturas, equipamiento, etc. Estas condiciones permiten o dificultan la propagación de sonidos que armonizan y se identifican con el espacio o se oponen y discrepan, creando así un ambiente sonoro que incide en las cualidades arquitectónicas y urbanísticas percibidas. En la evaluación cualitativa se considera importante analizar las fuentes y el tipo de sonidos que emiten, y cómo son percibidos e impactan en la subjetividad.

La investigación sobre paisaje sonoro llevada a cabo por Zhang et al., focaliza la experiencia perceptual de usuarios de espacios urbanos y la variabilidad espaciotemporal, con inclusión de aspectos físicos, sociales, culturales, psicológicos y arquitectónicos. En sus conclusiones muestran el efecto de cuatro dimensiones del paisaje sonoro -relajación, comunicación, espacialidad y dinámica-, sobre el confort acústico en espacios públicos urbanos abiertos (Zhang et al., 2018). En la misma línea de investigación, Grijalba Obando y Paül Carril verifican la dependencia entre los patrones de comportamiento espaciotemporal y sugieren que su variabilidad podría influir en la experiencia sonora de la ciudad latinoamericana. Concluyen que el paisaje sonoro debe formar parte de los procesos de planeamiento urbano y recomiendan considerar los parques urbanos y áreas verdes, no solo por motivos ambientales, sino con e fin de proveer a la ciudad y sus habitantes, de paisajes sonoros sanos y equilibrados. Asimismo, recomiendan para futuros estudios, ampliar la escala y profundizar sobre la relación entre el espacio público abierto y su paisaje sonoro resultante (Grijalba Obando y Paül Carril, 2018).

Es necesario señalar que la percepción del ambiente está integrada por los estímulos que llegan a través de los sentidos en su totalidad y constituyen la vivencia de entorno. En la focalización de la percepción auditiva, si bien interesa conocer cómo el individuo y la sociedad en su conjunto entienden el entorno acústico, lo registran e interpretan, se plantea el análisis de los estímulos sonoros integrados con otros estímulos presentes en el medio: los sonidos, la luz - los colores-, los olores, las texturas, el calor, el viento, etc.

\subsection{Relevamiento de las condiciones físicas y sonoras}

La plaza se desarrolla en una manzana o cuadra, de aproximadamente $2 \mathrm{Ha}$, con parte de su superficie con piso artificial y caminerías de hormigón alisado y aproximadamente dos tercios de su superficie de terreno natural, cubierto de césped o tierra. Presenta una importante masa arbórea estimada en 160 árboles, con un volumen aproximado de $14.000 \mathrm{~m} 3$ de copa o follaje, que forman un importante volumen vegetal de la zona. El equipamiento consiste en bancos de madera y meta dispuestos a ambos lados de las caminerías y sectores con bancos comunitarios circulares de hormigón que fueron instalados en la última renovación del equipamiento realizada en el año 2010. Parte de la caminería se encuentra elevada sobre el nivel de vereda, definida por bordes de hormigón, con rampas y escaleras. Como ya se mencionó, en la primera remodelación se reemplazó el lago natural que la caracterizaba por uno artificial, de $0.70 \mathrm{~m}$ de profundidad, que recorre parte de la plaza (Figuras 7 y 8 )
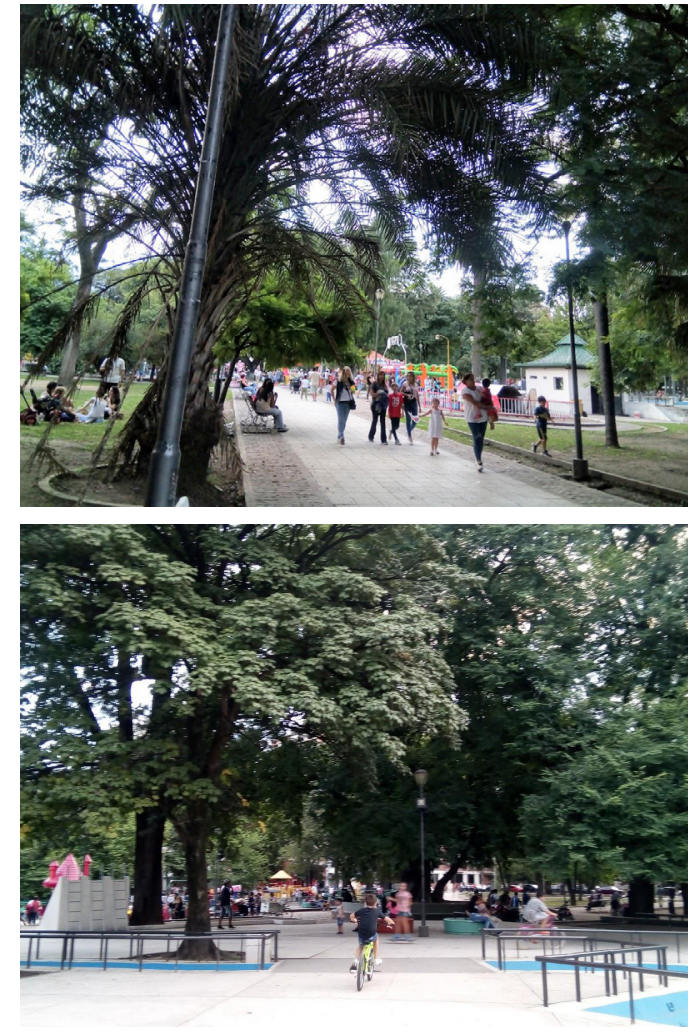

Figuras 7 y 8: Fotografía de la zona central y vista del lago artificial en su recorrido por algunos sectores de la plaza. Fuente: M.S. Cisterna (2019)

Los relevamientos permitieron conocer las zonas de la plaza donde se realizan las distintas actividades. En coincidencia con lo mencionado por los usuarios en las encuestas y entrevistas, los espacios son denominados según el equipamiento y algunas características formales. En base a los registros, es posible mencionar las siguientes zonas o sectores (Figura 9).

- Sector de Juegos infantiles

- Sector central con el legendario "árbol de la plaza" 
- Sector del mástil, con la explanada circular

- Sector del Monumento a Borges, frente al colegio Nacional B. Mitre

- Sector del lago con la explanada sobre calle 25 de mayo

\subsection{Medición de fuentes sonoras}

Los puntos de medición elegidos responden al objetivo de analizar el comportamiento general del área perimetra de borde y área central de la plaza. En base a las observaciones realizadas se pudo determinar el horario para las mediciones y toma de encuestas y entrevistas, el cual corresponde al de mayor concurrencia: los fines de semana, a partir de las 18:00 h hasta las 22:00 h. Los puntos de medición elegidos fueron las esquinas NorOeste y Sur-Este, el cruce de diagonales en el centro de la plaza y el sector frente al colegio Nacional.

Se realizaron seis visitas al lugar de estudio, durante un año. En cada una se llevaron a cabo observaciones, relevamientos fotográficos, entrevistas y relevamiento de fuentes sonoras. En el presente trabajo se muestran los datos obtenidos en las visitas donde se realizaron conjuntamente mediciones de las fuentes sonoras y aplicación de encuestas. Las mediciones se realizaron con decibelímetro TES SOUND LEVEL METER TES-1350A, durante los días domingo 31 de marzo y 7 de abril de 2019 y domingo 1 y viernes 6 de marzo de 2020, desde 18:00 h hasta 22:00 h. Se midieron las intersecciones de las cuatro vías de circulación vehicular y en el interior de la plaza en la intersección de las diagonales (en el centro) y en el sector de bancos donde se encuentra el monumento a Borges. El sonómetro se ubicó a $1.60 \mathrm{~m}$ del nivel de vereda, a una distancia de 2.00 metros del cordón de la calle y en forma manual se registraron las variaciones. Las mediciones se realizaron en periodos de tiempo de 60 minutos cada una, en los puntos seleccionados, resultando un total de cuatro horas. La lectura del medidor sonoro se realizó en compensación $\mathrm{A}$ en respuesta SLOW (1sec.) Level Range Lo 35-100 dB. Se realizaron también mediciones en compensación $\mathrm{C}$ para comparar ambos registros (Tabla 1). Al momento de los relevamientos se contaron más de 300 personas en la plaza, realizando diversas actividades: clase de gimnasia y de baile, grupo de jóvenes escuchando música, grupos sentados en los bancos conversando, personas realizando caminatas, niños en el sector de juegos $y$ también andando en bicicleta o rollers.

\begin{tabular}{|c|c|c|c|c|}
\hline \multicolumn{5}{|c|}{ Registros (dB) } \\
\hline \begin{tabular}{c} 
Lugar de medición \\
\hline $\begin{array}{c}\text { Av. Sarmiento y } \\
\text { Muñecas }\end{array}$
\end{tabular} & Min. & Max & Med. & Leq \\
\hline dBA & 72 & 84 & 78 & 75 \\
\hline dBA & 75 & 87 & 81 & \\
\hline En el centro de la plaza & Min. & Max & Med. & \\
\hline dBA & 65 & 75 & 70 & 68 \\
\hline dBA & 75 & 80 & 77.5 & \\
\hline 25 de mayo y Santa Fe & Min. & Max & Med. & \\
\hline dBA & 65 & 81 & 73 & 73 \\
\hline dBA & 80 & 89 & 84.5 & \\
\hline Monumento a Borges & Min. & Max & Med. & \\
\hline dBA & 62 & 67 & 64.5 & 64 \\
\hline
\end{tabular}

Tabla 1: Registros de Niveles de Intensidad Acústica Fuente: M. S. Cisterna (2020)
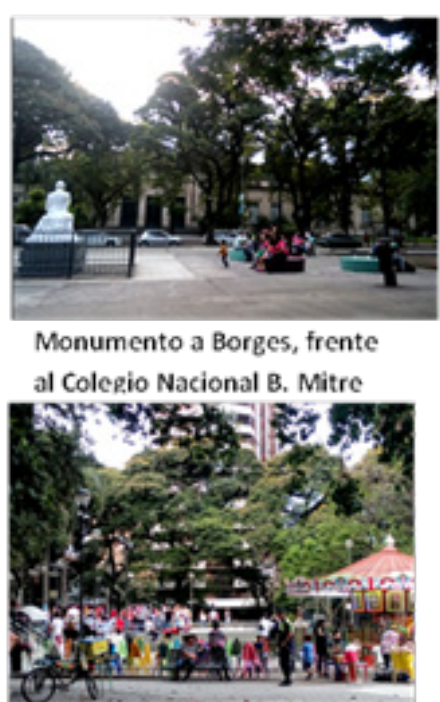

Juegos infantiles

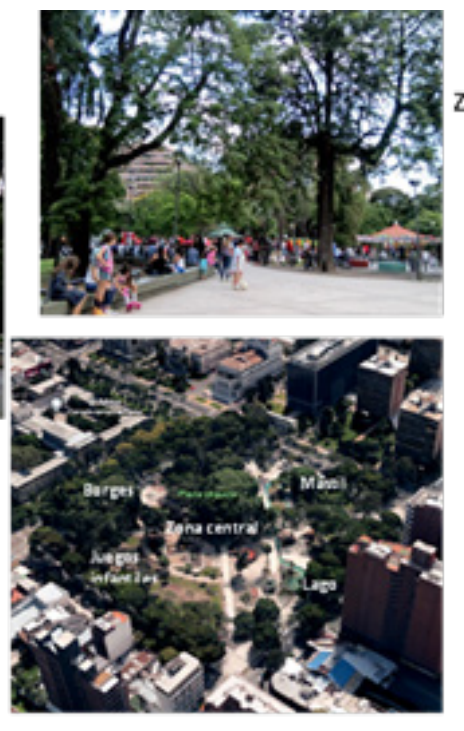

Zona central de la plaza

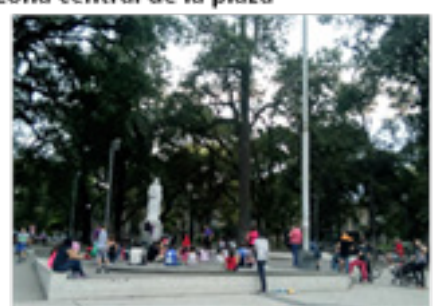

Explanada y mástil frente a

Avenida Sarmiento

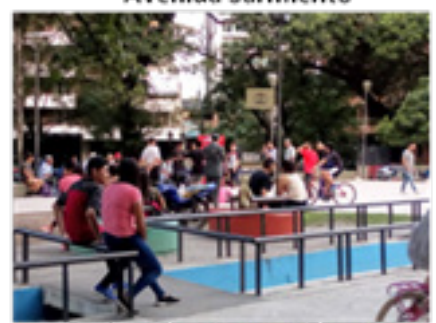

Explanada y lago artificial

Figura 9: Sectores registrados en el relevamiento. Fotografías de los distintos sectores de la plaza. Fuente: M.S. Cisterna (2019-2020) y Fotografía aérea

Fuente: https://www.google.com/intl/es-419/earth/ 
Los registros obtenidos están comprendidos en un rango de 62 a $84 \mathrm{~dB}(\mathrm{~A}) ; 75$ a $89 \mathrm{~dB}(\mathrm{C})$. Los registros ponderados A y ponderado $C$ muestran la presencia de sonidos de baja frecuencia; los mayores $\mathrm{dB}(\mathrm{C})$ se registraron en las zonas cercanas a las vías de circulación vehicular. Si bien estas frecuencias son percibidas en menor medida por el oído humano, forman parte del paisaje sonoro, y es importante considerarlas porque se detectan a través de los huesos esqueléticos, el oído, los armónicos, los sentidos táctiles o la resonancia en los órganos del cuerpo. Su detección aumenta la posibilidad de reacciones subjetivas como molestias, las cuales pueden contribuir de manera compleja a otros efectos biológicos y psicológicos de la señal, como describen Hatfield, Van Kamp y Job (2006). Los niveles sonoros equivalentes Leq(A) muestran que tanto en los sectores más cercanos a las vías de circulación como en el centro de la plaza los registros son superiores a $65 \mathrm{~dB}$ (recomendado por la OMS, valor adoptado por la normativa europea), por lo cual se pueden caracterizar como calles ruidosas. En el centro de la plaza los niveles registrados, Leq(A) $68 \mathrm{~dB}$, muestran que no constituye un lugar tranquilo, característica que no impactó negativamente en la valoración de los usuarios. El sector del monumento a Borges, frente al colegio Nacional, es el lugar donde se registraron niveles sonoros y Leq(A) levemente inferiores. A pesar de los altos niveles de sonidos registrados en la plaza, las personas entrevistadas y encuestadas no expresaron estar en inconfort. La mayoría de los usuarios valoraron positivamente el entorno sonoro de la plaza.

\section{Discusión y conclusiones}

La investigación permitió construir teoría sobre el paisaje sonoro de la Plaza Urquiza de Tucumán, a partir de registros y vivencias que los propios sujetos expresan en el contexto estudiado. Con la aplicación de la técnica de encuesta se recogieron datos, los cuales fueron analizados estadísticamente. Otros datos, obtenidos a través de observaciones, de entrevistas libres y entrevistas en profundidad a informantes clave, fueron analizados con el Método Comparativo Constante, el cual permite la integración de datos provenientes de la investigación empírica con la construcción de la Teoría de Base o Fundamentada. Con el uso de sonómetro se registraron los niveles sonoros en la plaza. Los datos cualitativos y cuantitativos obtenidos a través de la aplicación de diferentes técnicas, fueron procesados y analizados para la construcción de teoría sobre el paisaje sonoro. E trabajo realizado demuestra que la metodología aplicada permite el estudio de fenómenos complejos a través de la integración de diferentes técnicas, con énfasis en los aspectos cualitativos. A partir de procesos de análisis inductivo, se pueden inferir las siguientes conclusiones. S bien en las encuestas se registraron valoraciones positivas sobre el ambiente sonoro de la plaza, es importante señalar que, de las expresiones vertidas por los usuarios en las preguntas abiertas y en las entrevistas, se desprende la vivencia de un paisaje sonoro redundante y monótono. La mayoría de las personas reconoce el ruido del tránsito vehicular como la fuente de mayor presencia. Pocos usuarios (7\%) identificaron otras fuentes, como e canto de los pájaros, el sonido del viento en los árboles o de algunos insectos. Las personas que concurren al lugar se encuentran principalmente con un sonido de fondo que, debido a su intensidad, cobra protagonismo y dificulta la percepción de otras fuentes, siendo la música de los juegos infantiles y de grupos de jóvenes que all concurren, las únicas variaciones percibidas. El resultado es un paisaje sonoro de baja calidad que poco tiene que ver con las potenciales configuraciones que brinda un espacio verde, donde es posible lograr un equilibrio entre las diferentes fuentes de sonidos naturales, humanos y tecnológicos. En las entrevistas, los usuarios utilizaron expresiones relacionadas a la contención que les brinda el paisaje sonoro de la plaza, e incluso manifestaron su deseo de mantenerlo sin cambios. Estas expresiones se relacionan con el concepto referido al uso del sonido como "audioanalgésico" (Schafer, 1977), una forma de usar el sonido como un muro que bloquea el diálogo interno, tapa las emociones y también como una forma de distracción. Por otro lado, las referencias que hicieron los usuarios al ruido del tránsito como un sonido agradable que "da vida a la ciudad", evidencia que el mismo se asocia con el movimiento y la actividad continuos. Muy pocos se refirieron a la búsqueda de silencio para la lectura o el diálogo, o simplemente para escuchar los sonidos de la naturaleza. En relación a los sonidos urbanos, Truax señala que escuchar es una interfaz entre el individuo y el entorno y constituye un conjunto de habilidades que parecen estar deteriorándose. Atribuye tal situación al medio urbano tecnologizado, tanto por la exposición al ruido que causa pérdida auditiva y estrés fisiológico, como por la proliferación de sonidos altamente redundantes y básicamente poco interesantes, con poca información, que no fomentan la escucha sensible (Truax, 1984). Los espacios verdes en las ciudades brindan la posibilidad de mejorar la calidad de vida, disminuyen los niveles de dióxido de carbono $\mathrm{CO} 2$ del aire, detienen partículas de polvo, con su sombra protegen de la radiación solar, mejoran los niveles de humedad y constituyen el hábitat adecuado para muchas especies de aves que suman una fuente sonora natural que enriquece su paisaje sonoro. Un ejemplo de espacio acondicionado para propiciar el hábitat de las aves lo constituye el "Jardín encantado" en San Francisco Colombia, con más de 27 especies de colibríes. Nuestra ciudad actual, con sus fuentes sonoras artificiales y el incremento de los sonidos tecnológicos está expulsando a las aves, alterando sus ciclos reproductivos guiados por sus cantos, ha perdido parte de sus especies arbóreas nativas y se aleja cada vez más de ser el "Jardín de la República", como ha sido denominada históricamente. En el transcurso de la investigación han surgido nuevas ideas y temas de análisis - el arbolado como modificador del paisaje sonoro, la interacción del paisaje sonoro de la plaza con las construcciones circundantes, la incorporación de "objetos sonoros" en el diseño de los espacios públicos - temas que forman parte de la etapa de investigación actualmente en desarrollo.

\section{Recomendaciones}

El paisaje sonoro de la plaza podría beneficiarse, principalmente, de un diseño que incorpore objetivos en relación a la calidad del ambiente sonoro que se desea crear. Sería favorable permitir la escucha equilibrada de diversas fuentes, con sectores donde exista la posibilidad 
de aislarse de los sonidos del tránsito vehicular. La plaza cuenta con una importante masa arbórea, la cual puede ser el hábitat para diversas especies de aves y poblar así, con sus cantos el lugar. Esto permitiría contar con una fuente sonora natural. El lago artificial que recorre parte de su superficie cobraría importancia si se sumara el sonido del agua en movimiento, y tendría un impacto positivo en la percepción del ambiente. La convivencia de grupos que realizan diferentes actividades le otorga una dinámica interesante a la plaza. Los sectores donde se llevan a cabo deberían contar con un acondicionamiento acústico acorde a las mismas, y se podrían disponer elementos absorbentes para mejorar la calidad sonora y también pantallas estratégicamente ubicadas para disminuir la interferencia entre las diversas actividades sin obstaculizar las visuales. Las plazas de las ciudades constituyen un importante ámbito de convivencia; cumplen una importante función de espacio para la interacción social entre las personas y también con otras especies con las cuales compartimos el planeta. Los diseñadores tienen la posibilidad, y tal vez la obligación, de lograr una convivencia equilibrada en la cual se recupere la capacidad de escuchar y disfrutar de los sonidos, capacidad que se ha visto deteriorada por el avance de una ciudad que muchas veces resulta deshumanizante. Un diseño del espacio que considere la incorporación de pautas para mejorar el paisaje sonoro redundaría en un paisaje polifónico, heterogéneo y a la vez equilibrado, de mayor calidad acústica, con prevalencia de diversas fuentes, con el ruido del tránsito vehicular mitigado para disminuir la interferencia y el enmascaramiento sobre las otras fuentes. Un paisaje sonoro de tales características tendría un impacto positivo en la sustentabilidad del hábitat y en la calidad de vida de los habitantes.

Cómo citar este artículo/How to cite this article: Cisterna, S., Maristany, A. y Gonzalo, G. (2021). Impacto del paisaje sonoro urbano desde el registro subjetivo de los usuarios. Abordaje metodológico-instrumental. Estoa. Revista de la Facultad de Arquitectura y Urbanismo de la Universidad de Cuenca, 10(20), 141-152. doi: https://10.18537/ est.v010.n020.a12

\section{Referencias bibliográficas}

Aletta, F., Oberman, T., Mitchell, A., Erfanian, M., Lionello M., Kachlicka M. y Kang J. (2019). Associations between soundscape experience and self-reported wellbeing in open public urban spaces: a field study. The Lancet, 394. https://doi.org/10.1016/S0140-6736(19)32814-4

Botteldooren, D., De Coensel, B. y De Muer, T. (2006). The temporal structure of urban soundscapes. Journal of sound and vibration, 292(1), 105-123. https://doi. org/10.1016/j.jsv.2005.07.026

Charmaz K. (2006). Constructing Grounded Theory. A Practical Guide Through Qualitative Analysis. SAGE.

Di Lullo, R. y Giobellina, B. (1996). La otra ciudad. Tucumán frente al 2000. FAU UNT.

Flick, U. (2007). Introducción a la investigación cualitativa. Ediciones Morata.

García, A. I. (2010). Contaminación acústica en la materia de música. Innovación y experiencias educativas, 27, 1-9.

Germán González, M. y Santillán, A. O. (2006). Del concepto de ruido urbano al de paisaje sonoro. Bitácora.

Glaser B. y Strauss, A. (1967). The Discovery of Grounded Theory: Strategies for Qualitative Research. Aldine.

Glaser, B. (1992). Basics of Grounded Theory Analysis. California, Sociology Press.

Grijalba Obando, J. A. y Paül Carril, V. (2018). La influencia del paisaje sonoro en la calidad del entorno urbano. Un estudio en la ciudad de Popayán (Colombia). Urbano, 21(38), 70-83. https://doi.org/10.22320/07183607.201 8.21.38.06

Hatfield, J., Van Kamp, I, y Job, R. F. S. (2006). Clarifying "soundscape": Effects of question format on reaction to noise from combined sources. Acta Acustica United with Acustica, 92(6), 922-928.

Hernández Sampieri, R. y Mendoza, C. (2018). Metodología de la investigación. Las rutas cuantitativa, cualitativa y mixta. Mc Graw Hill.

López Barrio, I. (2001). El significado del medio ambiente sonoro en el entorno urbano. Estudios geográficos, 62 (244), 447-466. https://doi.org/10.3989/egeogr.2001. i244.277

Maristany, A. R. (2016). Paisaje Sonoro Urbano "Soundwalk" como método de análisis integral. Revista Pensum, 2, 41-56.

Matthew, B., Miles, A., Huberman, M. y Saldaña, J. (2019) Qualitative Data Analysis: A Methods Sourcebook. SAGE Publications.

Paterlini, O. (2010). La centralidad urbana histórica en San Miguel de Tucumán: entre la conservación y la innovación. En M. Gutman (Ed.), Argentina: persistencia 
$y$ diversificación, contrastes e imaginarios en las centralidades urbanas, (pp. 55-96). OLACCHI.

Schaeffer, P. (2003). Tratado de los objetos musicales. Alianza Editorial.

Schafer, R. M. (1977). The Tuning of the World (The Soundscape). Arcana Editions.

Schafer, R. M. (1976). El mundo del sonido. Los sonidos del mundo. Publicación Mensual de la UNESCO. EI Correo. XXIX. Place de Fontenoy.

Strauss, A. y Corbin, J. (1990). 1st Edition. Basics of Qualitative Research- Techniques and Procedures for Developing Grounded Theory. Sage.

Truax, B. (1984). Acoustic Communication. Simon Fraser University.

Truax, B. (1999). Handbook of Acoustic Ecology. (CDROM). Cambridge Street.

Zapata Cardona, G. A. y Cardona Restrepo, J. D. (2020) Relaciones entre el paisaje sonoro y la educación patrimonial: hacia el desarrollo de la inteligencia territorial. Trilogía Ciencia Tecnología Sociedad, 12(22), 217-244. https://doi.org/10.22430/21457778.1559

Zhang, X., Ba, M., Kang, J., y Meng, Q. (2018). Effect of Soundscape Dimensions on Acoustic Comfort in Urban Open Public Spaces. Applied Acoustics, 133, 73-81. https://doi.org/10.1016/j.apacoust.2017.11.024 